\title{
Temperature and Precipitation Drive Elevational Patterns of Microbial Beta Diversity in Alpine Grasslands
}

\section{Xiaoqin Yang}

Institute of Tibetan Plateau Research, Chinese Academy of Sciences

\section{Yue Li}

China University of Geosciences

\section{Bin Niu}

Institute of Tibetan Plateau Research, Chinese Academy of Sciences

\section{Qiuyu Chen}

Institute of Tibetan Plateau Research, Chinese Academy of Sciences

\section{Yilun $\mathrm{Hu}$}

Institute of Tibetan Plateau Research, Chinese Academy of Sciences

\section{Yibo Yang}

Institute of Tibetan Plateau Research, Chinese Academy of Sciences

\section{Lili Song}

Institute of Tibetan Plateau Research, Chinese Academy of Sciences

\section{Jianjun Wang}

Nanjing Institute of Geography and Limnology

Gengxin Zhang ( $D$ zhangg@itpcas.ac.cn )

Institute of Tibetan Plateau Research, Chinese Academy of Sciences https://orcid.org/0000-00019245-1981

\section{Research Article}

Keywords: soil microbes, elevational gradient, temperature, precipitation, LCBD, community composition

Posted Date: July 7th, 2021

DOI: https://doi.org/10.21203/rs.3.rs-662462/v1

License: (c) (i) This work is licensed under a Creative Commons Attribution 4.0 International License. Read Full License 


\section{Abstract}

Understanding the mechanisms underlying biodiversity patterns is a central issue in ecology, while how temperature and precipitation jointly control the elevational patterns of microbes is understudied. Here, we studied the effects of temperature, precipitation and their interactions on the alpha and beta diversity of soil archaea and bacteria in alpine grasslands along an elevational gradient of 4,300-5,200 $\mathrm{m}$ on the Tibetan Plateau. Alpha diversity was examined on the basis of species richness and evenness, and beta diversity was quantified with the recently developed metric of local contributions to beta diversity (LCBD). Typical alpine steppe and meadow ecosystems were distributed below and above 4,850 m, respectively, which was consistent with the two main constraints of mean annual temperature (MAT) and mean annual precipitation (MAP). Species richness and evenness showed decreasing elevational patterns in archaea and nonsignificant or U-shaped patterns in bacteria. The LCBD of both groups exhibited significant U-shaped elevational patterns, with the lowest values occurring at 4,800 $\mathrm{m}$. For the three diversity metrics, soil pH was the primary explanatory variable in archaea, explaining over $20.1 \%$ of the observed variation, whereas vegetation richness, total nitrogen and the K/Al ratio presented the strongest effects on bacteria, with relative importance values of $16.1 \%, 12.5 \%$ and $11.6 \%$, respectively. For the microbial community composition of both archaea and bacteria, the moisture index showed the dominant effect, explaining $17.6 \%$ of the observed variation, followed by MAT and MAP. Taken together, temperature and precipitation exerted considerable indirect effects on microbial richness and evenness through local environmental and energy supply-related variables, such as vegetation richness, whereas temperature exerted a larger direct influence on LCBD and the community composition. Our findings highlighted the profound influence of temperature and precipitation interactions on microbial beta diversity in alpine grasslands on the Tibetan Plateau.

\section{Introduction}

Spatial patterns of biodiversity are increasingly well documented [1]; however, the mechanisms underlying these patterns remain unclear [2]. Climate factors have been identified as the main drivers shaping diversity gradients [3]. For instance, a positive correlation between temperature and species richness has been found along latitudinal [4] and elevational gradients [5-7]. The main hypotheses explaining spatial diversity gradients are (1) the metabolic theory of ecology, relating higher mutation and speciation rates towards warmer areas [8], and (2) the productivity-diversity hypothesis, focusing on the positive effect of resources on species coexistence and diversity [9]. Furthermore, precipitation has been reported to be the main driver of biodiversity [10], which supports the water availability hypothesis, focusing on direct or indirect (via effects on productivity and soil environmental properties) constraints on biodiversity. For instance, precipitation constrains aboveground vegetation and soil moisture across bioclimatic zones, which indirectly influences species physiology [11], structure [12] and their interactions [13]. Thus, warmer and wetter conditions increase productivity, which can further influence biodiversity [14]. 
Elevational gradients in which climatic conditions vary greatly over a short geographical distance provide a 'natural laboratory' for testing the joint influence of temperature and precipitation on biodiversity [1516]. With increasing elevation, temperature generally decreases, and precipitation shows various elevational patterns, which are linked to diversity patterns [17-19]. Biodiversity is limited by drought or low temperature at lower or higher elevations, respectively [20-22]. The warmest-wettest conditions at intermediate elevations may predict hump-shaped elevational diversity patterns [14]. For instance, the bacterial [23] community has been reported to exhibit a hump-shaped elevational diversity pattern driven by temperature, in which precipitation follows a hump-shaped pattern as elevation increases. However, bacterial richness shows a U-shaped elevational pattern driven by mean annual temperature (MAT) and mean annual precipitation (MAP), in which decreasing and increasing elevational patterns are observed of temperature-precipitation [24]. Thus, it remains unclear how temperature and precipitation jointly control microbial biodiversity elevational patterns.

Here, we intensively reviewed elevational richness patterns for soil microbes under different temperature and precipitation combinations (Figs. S1, S2, Table S1). We further examined the temperature and precipitation effects on microbial biodiversity at 10 elevations covering alpine steppe and meadow vegetation along an elevational gradient (4,300-5,200 m) on the Tibetan Plateau (Figs. 1a, b). We projected MAT and MAP at sampling sites in a Whittaker biome plot to show how plant biomes are distributed in association with long-term precipitation and temperature [25]. We collected climate data with a high temporal and spatial resolution from 2007 to 2013. The grass line occurs at approximately $5,200 \mathrm{~m}$, which is sensitive to climate change. The temperature decreased, and precipitation followed a hump-shaped elevational pattern (Fig. S5). Thus, the elevational gradient provides an invaluable natural laboratory for testing whether temperature and precipitation jointly influence microbial biodiversity patterns. Herein, we aimed (1) to explore microbial biodiversity elevational patterns, (2) to estimate the joint impact of temperature and precipitation on microbial biodiversity, and (3) to evaluate the direct and indirect effects of temperature and precipitation on microbial biodiversity. We hypothesized that microbial biodiversity would exhibit hump-shaped or U-shaped elevational patterns, which may be strongly influenced by temperature, precipitation and their interactions in direct and indirect ways (Fig. 1c).

\section{Materials And Methods}

\subsection{Site description}

We examined a $900 \mathrm{~m}$ elevational gradient (4,300-5,200 $\mathrm{m}$ at $100 \mathrm{~m}$ vertical intervals) in the Nyainqentanglha Mountains ( $\left.30^{\circ} 18^{\prime}-30^{\circ} 32^{\prime} \mathrm{N}, 90^{\circ} 03^{\prime}-91^{\circ} 52^{\prime} \mathrm{E}\right)$ in Damxung County, Central-East Tibetan Plateau. The climate of the region is semiarid, with a growing season of 5 months from May to September. MAT is $2.1^{\circ} \mathrm{C}$, and MAP is $479 \mathrm{~mm}$. The precipitation in the growing season accounts for $90 \%$ of the total precipitation. Stipa capillacea and Stipa purpurea are dominant at low elevations of 4,300-4,500 m (alpine steppe ecotone), while Kobresia pygmaea is the dominant species at high elevations of 4,950-5,100 m (alpine meadows ecotone) [22], and mid-elevations are occupied by an ecotone of alpine steppe and meadow ecosystems. 
We set up eight HOBO weather stations (Onset Inc., Bourne, MA, USA) at 4,300, 4,400, 4,500, 4,650, 4,800, $4,950,5,100$, and $5,200 \mathrm{~m}$ and recorded air temperature and precipitation at 1-hour intervals. We collected climate data with a high temporal and spatial resolution from 2007 to 2013 . At 4,600, 4,700, 4,900 and $5,000 \mathrm{~m}$, temperature and precipitation were predicted from the data of the eight weather stations using a linear model. We calculated 13 temperature and 8 precipitation variables (Table S3), such as MAT and MAP as the long-term measurement for temperature and precipitation; precipitation seasonality (coefficient of variation) (PSCV), which is the ratio of the standard deviation of the monthly total precipitation to the mean monthly total precipitation [26]. To characterize the joint influence of temperature and precipitation on the microbial community, we calculated the ratio of growing season precipitation to the total accumulated temperature above $5^{\circ} \mathrm{C}$ (GSP/AccT) [22], which could serve as a substitute for the climate moisture index [27]. Statistical dependence between the climate variables was assessed using Spearman's rank correlation coefficients. Except for MAT, MAP and GSP/AccT, all the other pairwise Spearman's rank correlations were less than 0.75 . Thus, seven climate variables were retained, including MAT, the minimum temperature of the coldest month (MTCM), GSP/AccT, MAP, the precipitation of the driest month (PMD), PSCV and the mean annual coldest temperature $\left(T_{\text {min }}\right)$.

\subsection{Soil sample collection}

The results obtained from an adequate number of sample replications are more accurate for revealing the community distribution at each elevation. In August 2013, nine nested samples (10 cm depth) were collected at distances of 1 and $10 \mathrm{~m}$ at each elevation. Five soil cores were randomly taken from each site using a soil sampler tube ( $5 \mathrm{~cm}$ in diameter) and were then mixed as a composite sample. At each elevation, nine replications were performed. In total, there were 89 samples (we removed one sample collected at $10 \mathrm{~m}$ above $5,000 \mathrm{~m}$ because of contamination) collected over the whole elevational gradient. A total of $250 \mathrm{~g}$ of soil was sieved through a $2 \mathrm{~mm}$ mesh, and subsamples (approximately $50 \mathrm{~g}$ ) were transferred to plastic bags and placed into a $-80^{\circ} \mathrm{C}$ refrigerator for soil bacterial community analysis. The remaining soil (approximately $200 \mathrm{~g}$ ) was kept at $4{ }^{\circ} \mathrm{C}$ for soil property measurement.

\subsection{Environmental variables}

Soil samples were dried naturally at room temperature. Then, soil water content (SWC), pH, total nitrogen (TN), total phosphorus (TP), total organic carbon (TOC), soluble organic carbon (DOC) and soluble organic nitrogen (DON) were determined as potential explanatory variables of biodiversity. Briefly, $5 \mathrm{~g}$ soil samples were dried at $105^{\circ} \mathrm{C}$ for 12 hours, and SWC was calculated based on weight loss before and after drying. Five grams of soil was added to distilled water (soil:water =1:2.5), after which the mixture was stirred for $1 \mathrm{~min}$ and sat for $30 \mathrm{~min}$, and the $\mathrm{pH}$ was determined with a pH metre (Sartorius PB-10, Germany). TN was determined using a modified Kjeldahl method [28] and quantified with an elemental analyser (Vario Macro Cube, Elementar, Germany). TOC, DOC and DON were measured with a TOC analyser (TOC-VCPH, Shimadzu, Japan). TP was measured using the molybdenum blue method [29].

We measured soil geochemical elements and calculated the weathering index to evaluate its influence on biodiversity. Raw soil samples were digested by pressurized acid digestion using a mixture of $\mathrm{HNO}_{3}$ and 
$\mathrm{HF}$ in a Teflon digestion vessel [30]. Specifically, 20-30 mg soil samples were added to the vessel with 1 $\mathrm{ml} \mathrm{HNO}{ }_{3}$ and $1 \mathrm{ml} \mathrm{HF}$, and the mixture was then placed in an oven at $190^{\circ} \mathrm{C}$ for 24 hours. The cooling solutions were evaporated until they were nearly dry on a hot plate at $150^{\circ} \mathrm{C}$, after which $1 \mathrm{ml} \mathrm{HNO}_{3}$ was added, and the steps were repeated once. Next, $2 \mathrm{ml} \mathrm{HNO}_{3}$ and $3 \mathrm{ml}$ ultrapure water were added to the residue, and the mixture was heated at $150^{\circ} \mathrm{C}$ for 24 hours. Finally, the digestant was diluted to $50 \mathrm{ml}$ using ultrapure water. Metal elements, including $\mathrm{Ca}, \mathrm{Mg}, \mathrm{Fe}, \mathrm{Al}, \mathrm{K}, \mathrm{Na}, \mathrm{Ba}, \mathrm{Mn}, \mathrm{Ti}$ and $\mathrm{P}$, were measured using inductively coupled plasma optical emission spectroscopy (Leeman Labs, USA). Principal component analysis (PCA) was applied to extract the two principal component axes of the metal elements. The chemical index of alteration $\left(\mathrm{CIA}=\left[\left(\mathrm{Al}_{2} \mathrm{O}_{3}\right) /\left(\mathrm{Al}_{2} \mathrm{O}_{3}+\mathrm{CaO}+\mathrm{K}_{2} \mathrm{O}+\mathrm{Na}_{2} \mathrm{O}\right)\right] * 100\right)$ [31] and the $\mathrm{Ti} / \mathrm{Al}, \mathrm{Na} / \mathrm{Al}, \mathrm{K} / \mathrm{Al}$ and $\mathrm{Na} / \mathrm{K}$ ratios were calculated as indicators of weathering.

The normalized difference vegetation index (NDVI) is an indicator that reflects the growth state and coverage of vegetation. We downloaded Landsat 8 remote sensing image data (https://earthexplorer.usgs.gov/), applied ENVI 5.1 software for radiation calibration and atmospheric correction, and calculated the NDVI of the sampling site vegetation community through the band math module. The aspect and slope of the sampling sites were extracted from SRTMDEM 90 m-resolution raw elevation data (http://www.gscloud.cn/home) as potential explanatory variables of biodiversity. For the evaluation of the vegetation community, we established a $1 \mathrm{~m} \times 1 \mathrm{~m}$ quadrat at each elevation and recorded species present and the number of individuals of each species. Then, vegetation richness, the Shannon index and evenness were calculated.

\subsection{Community analyses}

Total soil DNA was extracted from $0.5 \mathrm{~g}$ soil subsamples by using the FastDNA® SPIN kit for soil (MP Biomedicals, USA). DNA extraction efficiency was determined by agar gel electrophoresis, and the obtained DNA concentration and purity were detected with a NanoDrop 2000c UV-Vis spectrophotometer (Thermo Fisher Scientific, USA). We used the 515F (5'-GTGCCAGCMGCCGCGGTAA-3') and 806R (5'GGACTACHVGGGTWTCTAAT-3') primers to amplify the 16S rRNA gene V4 region [32]. The PCR amplification steps were as follows: predenaturation at $94^{\circ} \mathrm{C}$ for $3 \mathrm{~min}$, denaturation at $94^{\circ} \mathrm{C}$ for $30 \mathrm{~s}$, annealing at $56^{\circ} \mathrm{C}$ for $30 \mathrm{~s}$, elongation at $72^{\circ} \mathrm{C}$ for $45 \mathrm{~s}$ (28 cycles) and elongation for 5 min until the temperature dropped to $4^{\circ} \mathrm{C}$. After the PCR products were purified by gel cutting, double-stranded DNA was quantified accurately by using PicoGreen (Invitrogen, Carlsbad, California). Then, the sequencing template was prepared by using the lon PGMTM Template 0T2 400 Kit (Life Technologies, USA). Sequencing data were derived via the lon Torrent PGM (Life Technologies, USA) approach [33]. All sequences were converted to FASTQ format using SAMtools [34]. Then, standardization processing was performed on a Linux system terminal. The sequences were processed with QIIME (v1.8). UCHIME was used to remove chimaeric sequences, and UCLUST was used to divide operational taxa with $97 \%$ similarity into operational taxonomic units (OTUs). The representative sequences of each OTU were then compared with data from the Ribosomal Database Project (RDP) for systematic classification [35]. Across all soil samples, we obtained a total of 2,904,266 high-quality sequences, with 32,632 sequences 
per sample on average. The raw data were deposited in the NCBI Sequence Read Archive database, and the project ID is PRJNA734468.

\subsection{Literature analysis}

We searched the data in the Web of Science (1990-September 2020) using the combined keywords ('altitude' or 'elevation') and ('diversity' or 'richness') and ('micro*'). We included studies on soil microbes such as archaea, bacteria and fungi involving $\geq 3$ sampling sites on each elevational gradient. We further compiled the elevational patterns of richness and then classified those patterns as significantly decreasing (D), increasing $(\mathrm{I})$, hump-shaped $(\mathrm{H})$, U-shaped $(\mathrm{U})$ and nonsignificant $(\mathrm{N})$ according to the results of the analysed papers [36].

\subsection{Statistical analyses}

The alpha and beta diversities of a community represent different facets of biodiversity driven by different environmental factors. We thus calculated species richness and Pielou's evenness [37] to represent microbial alpha diversity and local contributions to beta diversity (LCBD) [38] and the community composition to represent beta diversity. Species richness is the number of species in the ecosystem, and evenness represents the extent of evenly distributed species [36, 39]. We used Pielou's evenness because it is a good measure of the community distribution of relative abundance [40].

LCBD values quantify the relative contributions of individual sites to total beta diversity [38]. A high LCBD value for a site indicates that the site harbours a unique community composition. We computed LCBD values by using Hellinger-transformed abundance data and the LCBD.comp function by using adespatial (v0.3-8). Variations in community composition (beta diversity) [41] are a measure of differences in the microbial community composition between pairwise sites [42]. Principal coordinate analysis (PCoA) based on the community Bray-Curtis distance metric and PERMANOVA (Adonis statistic) was used to test the community composition along elevational gradients in the 'vegan' package (V 2.5.6).

To explore the elevational patterns of microbial richness, evenness and LCBD, we used linear or quadratic model. The more appropriate model was selected based on the lower value of Akaike's information criterion (AIC) [43]. Before evaluating the influence of different explanatory variables with different measurement units on microbial biodiversity, all variables were Z-score transformed. We conducted random forest analysis to estimate the relative importance of individual variables to community biodiversity using the ggRandomForests (V2.0.1) package [44]. A Mantel test was used to test the correlation between the microbial community and environmental variables. We classified all variables into three groups: climate variables, such as temperature and precipitation; local factors, such as soil physicochemical properties and the weathering index; and energy variables, such as vegetation, soluble carbon and nitrogen. We applied variation partitioning analysis (VPA) to identify the contributions of climate, energy and local variables (Table S4) to explaining microbial biodiversity. Before the analysis, collinear variables were removed by backward selection. 
We used structural equation models (SEMs) to test and quantify the direct and indirect effects of temperature and precipitation on microbial biodiversity. We ran all alternative models based on the full model (Fig. 1c) using AIC and model fit statistics [45]. We chose the final model with the lowest AIC value, and the detailed modelling fit indices are presented in Table S6. In addition, suitable model fits were evaluated on the basis of the AIC value, a nonsignificant $\chi^{2}$ test $(P>0.05)$, the comparative fit index (CFI> $0.95)$ and the standardized root mean squared residual (SRMR $<0.05)$. We ran the SEMs using the lavaan package (V0.6.5) [46], which provides multiple latent variable models to explain the underlying relationships in the models. All statistical analyses and plots were conducted in R statistical software V3.6.1 by using the packages vegan V2.5.6, ggplot2 V3.2.1, and corrplot V0.84.

\section{Results}

\subsection{Literature analysis of richness}

We compiled the elevational patterns of precipitation and species richness from the literature. Temperature shows a decreasing elevational pattern, whereas precipitation regimes differ across elevational gradients, which means that the species richness elevational patterns correspond to different precipitation patterns. Decreasing-increasing (D-I) and decreasing-hump-shaped (D-H) elevational patterns of temperature-precipitation accounted for $60.6 \%$ and $27.3 \%$, respectively, of 33 examined cases, whereas decreasing-decreasing (D-D) and decreasing-U-shaped (D-U) elevational patterns of temperatureprecipitation accounted for only a small proportion (6.1\%) (Fig. S1, Table S1). In total, $36.4 \%$ of the cases exhibited nonsignificant elevational patterns in all temperature-precipitation combinations, followed by decreasing (21.2\%) and hump-shaped (18.2\%) elevational patterns (Figs. S1, S2). Microbial richness showed hump-shaped elevational patterns in association with D-D elevational patterns of temperatureprecipitation. In $30.0 \%, 25.0 \%, 20.0 \%$ and $20.0 \%$ of cases, microbial richness exhibited decreasing, nonsignificant, hump-shaped and U-shaped elevational patterns, respectively, in association with D-I elevational patterns of temperature-precipitation. Microbial richness exhibited nonsignificant elevational patterns in association with D-U elevational patterns of temperature-precipitation. In $55.6 \%, 11.1 \%$ and $11.1 \%$ of cases, microbial richness exhibited nonsignificant, hump-shaped and U-shaped elevational patterns, respectively, in association with D-H pattern elevational patterns of temperature-precipitation.

Thus, there were no general elevational patterns of richness in association with different temperature and precipitation combinations. We further reviewed the main drivers shaping richness elevational patterns. In $54.5 \%$ of cases, local environmental variables were shown to be the most important drivers of species richness, followed by climate (30.3\%) and energy (6.1\%) variables (Table S1).

\subsection{Biodiversity patterns of microbial community}

Generally, alpha and beta diversity showed U-shaped and hump-shaped elevational patterns, respectively, at approximately 4,700-4,900 m. For species richness, the whole archaea and the phylum Crenarchaeota exhibited a significant $(P<0.01)$ decreasing pattern, while bacteria showed nonsignificant pattern (Figs. 2a, S8). Most bacterial phyla exhibited significant elevational patterns in richness (12 out of 14 
phyla), among which approximately $36 \%, 29 \%$ and $14 \%$ were U-shaped, hump-shaped and decreasing patterns $(P<0.05)$, respectively (Fig. S8). For species evenness, archaea and bacteria showed significant $(P<0.05)$ decreasing and U-shaped patterns with $\mathrm{R}^{2}$ values of 0.18 and 0.35 , respectively (Figs. $2 \mathrm{~b}, \mathrm{~S} 9$ ). Approximately $64 \%$ of phyla showed significant $(P<0.05)$ elevational patterns in evenness, among which approximately 29\%, 29\% and 7\% were U-shaped, hump-shaped and decreasing patterns, respectively (Fig. S9). For instance, Acidobacteria, Firmicutes and Proteobacteria exhibited significant U-shaped patterns; Gemmatimonadetes showed a significant hump-shaped pattern; and Crenarchaeota exhibited a significant decreasing elevational pattern (Fig. S9).

We observed consistent significant U-shaped LCBD-elevation relationships for archaeal $\left(\mathrm{R}^{2}=0.18, P<\right.$ $0.01)$ and bacterial $\left(R^{2}=0.38, P<0.01\right)$ communities (Fig. 2c). U-shaped patterns were also found for most bacterial phyla (12/14), such as Acidobacteria, Actinobacteria and Proteobacteria $(P<0.05)$ (Fig. S10). Interestingly, the lowest LCBD values for the whole microbial communities and their phyla occurred at approximately 4,700-4,900 m. The microbial community composition was mainly differentiated by elevation (Adonis statistic: $\mathrm{R}^{2}>0.11, P=0.001$; Figs. $2 \mathrm{~d}$, e).

\subsection{Underlying drivers of elevational biodiversity}

Typical alpine steppe and meadow ecosystems were distributed below and above 4,850 m, which was consistent with the two main constraints of MAT and MAP (Fig. 1a). The climate and vegetation ecotone also occurred at approximately 4,800-4,900 m in conceptual Whittaker biome plot (Fig. 1b). Climate, local and energy variables varied substantially along elevational gradients. For instance, MAT $\left(-1.9-2.84{ }^{\circ} \mathrm{C}\right)$, MAP (313-552 mm) and PSCV (134-141) showed significant (Pख0.05) decreasing, hump-shaped and Ushaped elevational patterns, respectively (Figs. S3, S5). Energy variables, such as the vegetation Shannon index (1.28-1.92), showed increasing and hump-shaped elevational patterns ( $P \otimes 0.05)$, while the local variable of soil pH showed a significant (P凶0.05) U-shaped elevational pattern (Fig. S3).

Soil $\mathrm{pH}$ was the most important explanatory variable, explaining $20.1 \%, 55.8 \%$ and $21.4 \%$ of the variation in archaeal richness, evenness and LCBD, respectively (Figs. 3a, b, c). Vegetation richness, TN and the $\mathrm{K} / \mathrm{Al}$ ratio had the strongest effects on bacterial richness, evenness and LCBD, with relative importance values of $16.1 \%, 12.5 \%$ and $11.6 \%$, respectively (Figs. $3 e, f, g$ ). The moisture index (GSP/AccT ratio) was the most important explanatory factor, explaining over $17.6 \%$ variation of microbial community composition represented by the first axis of PCoA (PCoA1), followed by MAT and MAP (Figs. 3d, $h$ ). Microbial richness, evenness and LCBD at the phylum level were driven by different variables. For richness, $\mathrm{T}_{\mathrm{min}}, \mathrm{PMD}, \mathrm{pH}$, vegetation richness and TP were the most important predictors, explaining $15.7 \%, 27.3 \%, 18.1 \%, 32.6 \%$ and $45.3 \%$ of the variation in Acidobacteria, Betaproteobacteria, Gemmatimonadetes, Firmicutes and Cyanobacteria, respectively (Fig. S16). For evenness, $\mathrm{T}_{\mathrm{min}}, \mathrm{pH}$ and slope were the primary drivers, explaining $21.5 \%, 48.7 \%$ and $56.8 \%$ of the variation in Deltaproteobacteria, Gammaproteobacteria and Crenarchaeota, respectively (Fig. S17). For LCBD, MTCM and slope had the strongest effects on Actinobacteria and Crenarchaeota, with relative importance values of $14.3 \%$ and $25.8 \%$, respectively (Fig. S18). 
Temperature and precipitation exerted considerable indirect effects on microbial richness and evenness through local and energy variables, whereas temperature directly influenced microbial LCBD and PCoA1. The total effects of temperature were larger than those of precipitation in explaining the variations in microbial evenness, LCBD and PCoA1 (Figs. 4, S19). Local and energy variables had direct effects on microbial alpha and beta diversity $(R>0.22)$ (Fig. 4). For archaea, the final SEMs explained $20.9 \%, 29.1 \%$, $37.8 \%$ and $65.1 \%$ of the variation in richness, evenness, LCBD and PCoA1 (Figs. 4a-d), respectively. Temperature had a larger indirect influence on microbial evenness, LCBD and PCoA1 than precipitation, with $\mathrm{R}$ values over 0.22 and 0.07 (Table S6). Temperature had direct effects on archaeal LCBD and PCoA1, with R values of 0.24 and $0.37(P \otimes 0.05)$, respectively (Figs. 4c, d). For bacteria, the final SEMs explained $15.1 \%, 54.7 \%, 67 \%$ and $78.5 \%$ of the variation in richness, evenness, LCBD and PCoA1 (Figs. 4eh), respectively. Temperature had a larger indirect influence on microbial alpha and beta diversity than precipitation, with $\mathrm{R}$ values over 0.2 and 0.07 (Table S6), respectively. Temperature had significant $(P \otimes 0.05)$ direct effects on bacterial LCBD and PCoA1, with R values over 0.39.

Such effects of climate, energy and local variables were further statistically supported by linear or quadratic regression analysis (Fig. S13), Mantel's test (Fig. S14) and variation partitioning analysis (Fig. S15). For instance, DON, MAT, TP and PMD were the most important drivers of archaeal richness, evenness, LCBD and PCoA1 ( $P \otimes 0.05$ ), with $\mathrm{R}^{2}$ values of $0.12,0.18,0.26$ and 0.42 , respectively. PSCV, the first PCA axis of metal variables (metal.pc1), MTCM and TP were the most important drivers of bacterial richness, evenness, LCBD and PCoA1, with $\mathrm{R}^{2}$ values of $0.05,0.30,0.51$ and $0.70(P \otimes 0.05)$, respectively (Fig. S13). The microbial communities were most significantly correlated with the GSP/AccT ratio ( $\mathrm{R}>$ $0.37, P<0.01)$ (Fig. S14). Variation partitioning analyses showed that the combined effects of climate, energy and local variables explained over $18 \%$ of the variation in microbial LCBD and PCoA1, whereas the effects of these three groups of variables explained only slightly over $3 \%$ of the variation in microbial richness and evenness. Climate variables showed pure effects larger than local and energy variables in explaining microbial LCBD and PCoA1 (Fig. S15).

\section{Discussion}

Temperature and precipitation have been suggested to shape soil microbial biodiversity along environmental gradients [47-48]; however, their joint influence on the elevational patterns of soil microbes has rarely been reported. Here, we tested the hypotheses that microbial alpha and beta diversity are mainly constrained by precipitation at low elevations but by low temperature at high elevations, resulting in hump-shaped and U-shaped elevational patterns associated with the combination of temperature and precipitation in alpine grasslands in semiarid regions. We found clear elevational patterns of species richness, evenness, LCBD and the community composition, which were primarily explained by the moisture index, representing the combination of temperature and precipitation. Furthermore, temperature had considerable direct and indirect effects on microbial alpha and beta diversity, while precipitation exerted relatively minor and indirect effects. Overall, these results supported our hypotheses and further suggested that the response of microbial alpha and beta diversity to 
temperature and precipitation may vary with elevation because of elevational shifts in temperature and precipitation limiting microbial growth.

\subsection{Elevational patterns of microbial community biodiversity}

Microbial alpha and beta diversity can provide different insights into elevational diversity patterns [36, 49]. Soil microbial alpha diversity has shown decreasing (34\%) or nonsignificant (33\%) elevational patterns in meta-analyses [50]; however, species richness showed nonsignificant (36.4\%) and decreasing $(21.2 \%)$ elevational patterns in our literature review, which was in line with $35 \%$ and $30 \%$ of the cases showing nonsignificant and decreasing elevational patterns, respectively [36]. These results demonstrated that a decreasing or nonsignificant pattern was probably a feature of the microbial alpha diversity elevational pattern [3]. Furthermore, the differences in the distribution of elevational diversity indicate the importance of considering habitat differences associated with different combinations of temperature and precipitation [21].

The declining elevational patterns of archaeal richness were consistent with the genes of soil ammoniaoxidizing archaea identified within the same elevational gradient [51], although the maximum diversity of soil archaea was found at mid-elevations [52]. Bacterial richness showed a nonsignificant elevational pattern in alpine grasslands, which was consistent with previous studies; for instance, $25.0 \%$ and $55.6 \%$ of the studies showed nonsignificant elevational patterns among D-D and D-H elevational patterns of temperature-precipitation, respectively. The diversity patterns of the dominant phyla contributed significantly to the whole community pattern; thus, U-shaped (36\%) and hump-shaped (29\%) patterns for bacterial phyla contributed to a nonsignificant pattern of whole bacterial richness. These phylum patterns were also in line with hump-shaped and U-shaped elevational patterns in places where precipitation followed a hump-shaped elevational pattern [7, 53]. However, the U-shaped elevational pattern of bacterial evenness contrasted with increasing, decreasing and nonsignificant patterns of stream bacterial evenness reported along multiple mountainsides across Asia and Europe [36]. When we scaled down to the phylum level, most of the phyla (> 58\%) showed significant U-shaped and hump-shaped elevational patterns of evenness, which have rarely been documented [15]. For LCBD, the whole archaeal and bacterial communities and their phyla exhibited a consistent U-shaped pattern, suggesting that such pattern is probably a general feature of the elevational pattern of microbial beta diversity $[15,54]$, mainly because of the greater uniqueness of the community composition at both ends of the elevational gradient [38]. The microbial community composition was significantly affected by elevation, which was in line with previous findings $[5,55,56]$.

\subsection{Underlying mechanisms of microbial biodiversity}

Despite the increasing attention given to the elevational biodiversity patterns of microbes, the joint effects of temperature and precipitation on microbial biodiversity patterns have not been well studied. Temperature and precipitation put restrictions on how many species can survive at different elevations; 
i.e., temperatures or precipitation levels determine the minimum and maximum niches within which a species can survive or restrict species productivity, which in turn limits the total number of individuals [57-58]. The alpha and beta diversity of flora and fauna along elevational gradients are commonly driven by temperature and precipitation in arid and semiarid ecotones or environments [59-60]. We found that the moisture index, representing the combination of temperature and precipitation, was the primary driver of the microbial community composition. To the best of our knowledge, this is the first report indicating that the interactions of temperature and precipitation exert a profound influence on microbial beta diversity along elevational gradients. Furthermore, the lowest values of the U-shaped LCBD pattern occurred at mid-elevations (4,700-4,900 m) (Fig. 2), where the relationship between the moisture index and LCBD showed a transition (Fig. S11). Thus, interactions of temperature and precipitation at midelevations may support more similar microbial communities, thereby shaping U-shaped elevational patterns of LCBD, which contrasts with findings showing that temperature alone drives the elevational patterns of stream bacteria, diatoms and macroinvertebrates along six elevational gradients [54]. One possible reason for the contrasting results between the two studies on microbes could be habitat differences. Furthermore, mid-elevations (4,700-4,900 m) are transition ecotones of soil nutrient availability and vegetation under a temperature-precipitation regime, which may also impact microbial beta diversity [61].

In our study, MAT and MAP were both found to be the main drivers of the soil microbial community composition, in accord with the metabolic theory of ecology [23-24], the productivity-diversity hypothesis and the water availability hypothesis [62]. This result was consistent with those of earlier reports on the stream microbial community composition driven by mean temperature and mean precipitation along elevational gradients in Norway, Spain and China [63]. However, precipitation was shown to be the primary factor shaping bacterial community structure along the same elevational gradient according to terminal restriction fragment length polymorphism analysis [64]. The microbial community composition is dependent on the long-term development of near-ground temperature and soil moisture regimes [19]. Thus, long-term (2007-2013) climate data with a high temporal and spatial resolution could precisely reveal the impacts of temperature and precipitation on the microbial community, which may explain the inconsistent results of different articles.

Climate has direct and indirect effects on microbial diversity [65]. However, temperature both directly and indirectly influences microbial alpha and beta diversity, which has rarely been reported $[49,66]$. With increasing temperature, vegetation richness increases and soil pH decreases, indirectly influencing microbial richness [67]. For microbial LCBD and the microbial community composition, the direct effects of temperature were dominant, mainly explained by temperature impacting microbial metabolism rates and growth rates [8]. The larger total effects of temperature than precipitation in explaining variations in microbial alpha and beta diversity were in line with reports of plant and animal diversity along an elevational gradient [68]. Precipitation can directly influence the microbial community through shifts in osmotic pressure and resource availability, which can induce responses related to microbial physiological stress, growth and metabolic activity [69]. Thus, precipitation deficits can limit soil microbe growth and activity by inhibiting the diffusion of nutrients and carbon substrates in soil samples [70]. Our results 
clearly indicated that precipitation exerted an indirect effect on microbial alpha and beta diversity via local environmental and energy supply-related variables, such as vegetation richness. Changes in precipitation or evapotranspiration influence vegetation types, soil pH and other soil characteristics, especially in arid and semiarid environments $[2,71]$; thus, precipitation has a considerable indirect impact on microbial alpha and beta diversity.

In addition to the main effects of temperature and precipitation, local and energy variables played a secondary role in shaping diversity patterns. Soil pH was the main driver of the richness, evenness and LCBD of archaea, which was in line with previous studies in soil bacteria [64, 72, 73]. However, the finding that soil pH had a minor impact on bacterial alpha and beta diversity was unexpected. Soil pH is an integrated functional index related to soil characteristics [74] that imposes physiological stress on individual growth and the microbial distribution [75]. Archaea inhabit more restricted ecological niches, which may make them more sensitive to soil $\mathrm{pH}$ variations along elevational gradients than bacteria [76]. Vegetation is the key driver of soil microbial diversity through the alteration of soil nutrient cycling [7778]. For instance, plant diversity exerts a substantial influence on soil bacterial beta diversity [79]. Vegetation richness was found to have the strongest impact on bacterial richness, which was in line with previous studies [80-81] and in accord with the productivity hypothesis, which emphasizes the positive effect of resources on species diversity $[9,58]$. Higher-diversity plant communities are composed of more diverse leaves and roots, and the release of more diverse root exudates can promote soil microbial diversity [82]. For instance, the diversity of soil bacteria peaks under conditions of high phosphorus availability but relatively low total carbon levels [83]. Vegetation richness showed significant positive relationships with TP and TOC (Fig. S4). Furthermore, vegetation richness and bacterial richness showed a nonlinear relationship (Fig. S12). These results indicated that energy variables were limiting factors for bacterial richness. The unexplained variations $(>85 \%)$ in microbial richness may have been caused by unmeasured environmental factors or biological factors [84]. It was further concluded that the elevational patterns of the alpha and beta diversity of microbial phyla were driven by distinct variables largely due to niche differentiation among taxa along elevational gradients [15]. For instance, the richness of Acidobacteria showed a decreasing elevational pattern and was strongly correlated with temperature, which was consistent with Bryant's findings [85].

\section{Conclusions}

Our literature analysis revealed that soil species richness is dominated by a nonsignificant elevational pattern, followed by significant decreasing patterns under different combinations of temperature and precipitation. Furthermore, archaeal richness and evenness exhibited decreasing elevational patterns, while bacterial richness and evenness showed nonsignificant and U-shaped elevational patterns, respectively. The LCBD values of both microbial groups and most phyla (12/14) showed significant Ushaped elevational patterns, and the lowest values were found at mid-elevations. The variations in species richness, evenness and LCBD were primarily explained by local environmental variables or energy variables, such as soil $\mathrm{pH}, \mathrm{K} / \mathrm{Al}, \mathrm{TN}$ and vegetation richness, while the community composition was most strongly explained by the moisture index, followed by MAT and MAP. Our results also highlighted that 
temperature exerted considerable direct and indirect effects on microbial alpha and beta diversity, while precipitation exerted relatively minor and indirect effects. Overall, these results suggested that temperature, precipitation and their interactions profoundly influence microbial beta diversity along elevational gradients in alpine grasslands in semiarid areas.

\section{Declarations}

\section{Conflict of Interest}

The authors declare that they have no conflicts of interest.

\section{Author Contributions}

Xiaoqin Yang: Conceptualization, Investigation, Validation, Data analyzation, Writing-original draft, Writing-review \& editing. Jianjun Wang: Conceptualization, Validation, Formal analysis and Methodology, Writing-review \& editing. Gengxin Zhang: Supervision,Resources, Funding acquisition, Conceptualization, Validation, Writing-review \& editing. Yue Li: Data analyzation. Yibo Yang: Data curation, Writing-review \& editing. Bin Niu, Qiuyu Chen, Yilun Hu, Lili Song: Writing-review \& editing. All co-authors participated in discussions and revised the manuscript.

\section{Acknowledgements}

We are grateful to Conghai Han for soil sample collection. This research was supported by grants from the National Natural Science Foundation of China $(41871066,41471055)$, the Strategic Priority Research Program (A) of the Chinese Academy of Sciences (XDA20050101), and the Second Tibetan Plateau Scientific Expedition and Research Program (STEP) (2019QZKK0503).

\section{References}

1. Gaston KJ (2000) Global patterns in biodiversity. Nature 405:220-227

2. Rahbek C, Borregaard MK, Colwell RK et al (2019) Humboldt's enigma: what causes global patterns of mountain biodiversity? Science 365:1108-1113

3. Peters MK, Hemp A, Appelhans T et al (2019) Climate-land-use interactions shape tropical mountain biodiversity and ecosystem functions. Nature 568:88-92

4. Zhou J, Deng Y, Shen L et al (2016) Temperature mediates continental-scale diversity of microbes in forest soils. Nat Commun 7:12083

5. Tian J, Wu B, Chen $\mathrm{H}$ et al (2017) Patterns and drivers of fungal diversity along an altitudinal gradient on Mount Gongga, China. J Soils Sediments 17:2856-2865

6. Duclos TR, DeLuca WV, King DI (2019) Direct and indirect effects of climate on bird abundance along elevation gradients in the Northern Appalachian Mountains. Divers Distrib 25:1670-1683 
7. Shen C, Gunina A, Luo Y et al (2020) Contrasting patterns and drivers of soil bacterial and fungal diversity across a mountain gradient. Environ Microbiol 22:3287-3301

8. Price CA, Weitz JS, Savage VM et al (2012) Testing the metabolic theory of ecology. Ecol Lett 15:1465-1474

9. Hurlbert AH, Stegen JC (2014) When should species richness be energy limited, and how would we know? Ecol Lett 17:401-413

10. Miyamoto $Y$, Nakano T, Hattori $M$ et al (2014) The mid-domain effect in ectomycorrhizal fungi: range overlap along an elevation gradient on Mount Fuji, Japan. ISME J 8:1739-1746

11. Lennon JT, Aanderud ZT, Lehmkuhl BK et al (2012) Mapping the niche space of soil microorganisms using taxonomy and traits. Ecology 93:1867-1879

12. Angel R, Soares MIM, Ungar ED et al (2010) Biogeography of soil archaea and bacteria along a steep precipitation gradient. ISME J 4:553-563

13. Treves DS, Xia B, Zhou J et al (2003) A two-species test of the hypothesis that spatial isolation influences microbial diversity in soil. Microb Ecol 45:20-28

14. McCain CM (2009) Global analysis of bird elevational diversity. Glob Ecol Biogeogr 18:346-360

15. Yeh CF, Soininen J, Teittinen A et al (2019) Elevational patterns and hierarchical determinants of biodiversity across microbial taxonomic scales. Mol Ecol 28:86-99

16. Hu A, Wang J, Sun $\mathrm{H}$ et al (2020) Mountain biodiversity and ecosystem functions: interplay between geology and contemporary environments. ISME J 14:931-944

17. McCain CM, Grytnes JA (2010) Elevational Gradients in Species Richness, Encyclopedia of Life Sciences. John Wiley \& Sons, Chichester

18. Lanzen A, Epelde L, Blanco F et al (2016) Multi-targeted metagenetic analysis of the influence of climate and environmental parameters on soil microbial communities along an elevational gradient. Sci Rep 6:28257

19. Frindte K, Pape R, Werner K et al (2019) Temperature and soil moisture control microbial community composition in an arctic-alpine ecosystem along elevational and micro-topographic gradients. ISME J 13:2031-2043

20. Gorban AN, Pokidysheva LI, Smirnova EV et al (2011) Law of the Minimum Paradoxes. Bull Math Biol 73:2013-2044

21. Kessler M, Kluge J, Hemp A et al (2011) A global comparative analysis of elevational species richness patterns of ferns. Glob Ecol Biogeogr 20:868-880

22. Wang Z, Luo T, Li R et al (2013) Causes for the unimodal pattern of biomass and productivity in alpine grasslands along a large altitudinal gradient in semi-arid regions. J Veg Sci 24:189-201

23. Nottingham AT, Fierer N, Turner BL et al (2018) Microbes follow Humboldt: temperature drives plant and soil microbial diversity patterns from the Amazon to the Andes. Ecology 99:2455-2466

24. Singh D, Lee-Cruz L, Kim WS et al (2014) Strong elevational trends in soil bacterial community composition on Mt. Halla, South Korea. Soil Biol Biochem 68:140-149 
25. Deshmukh A, Singh RA (2019) Whittaker biome-based framework to account for the impact of climate change on catchment behavior. Water Resour Res 55:11208-11224

26. Hijmans RJ, Cameron SE, Parra JL et al (2005) Very high resolution interpolated climate surfaces for global land areas. Int J Climatol 25:1965-1978

27. Fang J, Yoda K (1990) Climate and vegetation in China III water balance and distribution of vegetation. Ecol Res 5:9-23

28. Bremner JM (1960) Determination of nitrogen in soil by the Kjeldahl method. J Agr Sci 55:11-33

29. Shi Y, Li Y, Xiang X et al (2018) Spatial scale affects the relative role of stochasticity versus determinism in soil bacterial communities in wheat fields across the North China Plain. Microbiome $6: 27$

30. Yang Y, Fang X, Galy A et al (2016) Plateau uplift forcing climate change around $8.6 \mathrm{Ma}$ on the northeastern Tibetan Plateau: evidence from an integrated sedimentary Sr record. Palaeogeogr Palaeoclimatol Palaeoecol 461:418-431

31. Nesbitt HW, Young GM (1982) Early proterozoic climates and plate motions inferred from major element chemistry of lutites. Nature 299:715-717

32. Caporaso JG, Lauber CL, Walters WA et al (2011) Global patterns of 16S rRNA diversity at a depth of millions of sequences per sample. Proc Natl Acad Sci USA108:4516-4522

33. Van den Hoecke $S$, Verhelst J, Vuylsteke $M$ et al (2015) Analysis of the genetic diversity of influenza A viruses using next-generation DNA sequencing. BMC Genom 16:79

34. Li H, Handsaker B, Wysoker A et al (2009) The sequence alignment/map (SAM) format and SAMtools. Bioinformatics 25:2078-2079

35. Cole JR, Wang Q, Cardenas E et al (2009) The Ribosomal Database Project: improved alignments and new tools for rRNA analysis. Nucleic Acids Res 37:D141-D145

36. Wang J, Meier S, Soininen J et al (2017) Regional and global elevational patterns of microbial species richness and evenness. Ecography 40:393-402

37. Pielou EC (1966) Measurement of diversity in different types of biological collections. J Theor Biol 13:131-144

38. Legendre P, De Caceres M (2013) Beta diversity as the variance of community data: dissimilarity coefficients and partitioning. Ecol Lett 16:951-963

39. Roberts FS (2019) Measurement of Biodiversity: Richness and Evenness. Springer, Cham

40. Jost $L$ (2010) The relation between evenness and diversity. Diversity 2:207-232

41. Martiny JB, Eisen JA, Penn K et al (2011) Drivers of bacterial beta-diversity depend on spatial scale. Proc Natl Acad Sci USA 108:7850-7854

42. Wang J, Wu Y, Jiang $\mathrm{H}$ et al (2008) High beta diversity of bacteria in the shallow terrestrial subsurface. Environ Microbiol 10:2537-2549

43. Yamaoka K, Nakagawa T, Uno T (1978) Application of Akaikes information criterion (AIC) in evaluation of linear pharmacokinetic equations. J Pharmacokinet Pharmacodyn 6:165-175 
44. Lawrence RL, Wood SD, Sheley RL (2006) Mapping invasive plants using hyperspectral imagery and Breiman Cutler classifications (RandomForest). Remote Sens Environ 100:356-362

45. Grace JB, Anderson TM, Olff H et al (2010) On the specification of structural equation models for ecological systems. Ecol Monog 80:67-87

46. Rosseel Y (2012) Lavaan: an R package for structural equation modeling. J Stat Softw 48:1-36

47. Tedersoo L, Bahram M, Polme S et al (2014) Global diversity and geography of soil fungi. Science 346:1256688

48. Bahram M, Hildebrand F, Forslund SK et al (2018) Structure and function of the global topsoil microbiome. Nature 560:233-237

49. Zheng Y, Ji N, Wu B et al (2020) Climatic factors have unexpectedly strong impacts on soil bacterial $\beta$-diversity in 12 forest ecosystems. Soil Biol Biochem 142:107699

50. Looby Cl, Martin PH (2020) Diversity and function of soil microbes on montane gradients: the state of knowledge in a changing world. FEMS Microbiol Ecol 96:fiaa122

51. Yuan Y, Si G, Li W et al (2015) Altitudinal distribution of ammonia-oxidizing archaea and bacteria in alpine grassland soils along the south-facing slope of Nyqentangula mountains, central Tibetan Plateau. Geomicrobiol J 32:77-88

52. Singh D, Takahashi K, Park J et al (2016) Similarities and contrasts in the archaeal community of two Japanese mountains: Mt. Norikura compared to Mt. Fuji Microb Ecol 71:428-441

53. Shearer CA, Zelski SE, Raja HA et al (2015) Distributional patterns of freshwater ascomycetes communities along an Andes to Amazon elevational gradient in Peru. Biodivers Conserv 24:18771897

54. Wang J, Legendre P, Soininen J et al (2019) Temperature drives local contributions to beta diversity in mountain streams: stochastic and deterministic processes. Glob Ecol Biogeogr 29:420-432

55. Zhang Y, Cong J, Lu H et al (2015) Soil bacterial diversity patterns and drivers along an elevational gradient on Shennongjia Mountain, China. Microbiol Biotechnol 8:739-746

56. Sheng $Y$, Cong W, Yang $L$ et al (2019) Forest soil fungal community elevational distribution pattern and their ecological assembly processes. Front Microbiol 10:02226

57. Brown JH (2001) Mammals on mountainsides: elevational patterns of diversity. Global Ecol Biogeogr 10:101-109

58. Hawkins BA, Field R, Cornell HV et al (2003) Energy, water, and broad-scale geographic patterns of species richness. Ecology 84:3105-3117

59. Jankowski JE, Ciecka AL, Meyer NY et al (2009) Beta diversity along environmental gradients: implications of habitat specialization in tropical montane landscapes. J Anim Ecol 78:315-327

60. Sundqvist MK, Sanders NJ, Wardle DA et al (2013) Community and ecosystem responses to elevational gradients: processes, mechanisms, and insights for global change. Annu Rev Ecol Evol Syst 44:261-280 
61. Condit R, Engelbrecht BMJ, Pino D et al (2013) Species distributions in response to individual soil nutrients and seasonal drought across a community of tropical trees. Proc Natl Acad Sci USA 110:5064-5068

62. Wang J, Pan F, Soininen J et al (2016) Nutrient enrichment modifies temperature-biodiversity relationships in large-scale field experiments. Nat Commun 7:13960

63. Picazo F, Vilmi A, Aalto J et al (2020) Climate mediates continental scale patterns of stream microbial functional diversity. Microbiome 8:1-14

64. Yuan Y, Si G, Wang J et al (2014) Bacterial community in alpine grasslands along an altitudinal gradient on the Tibetan Plateau. FEMS Microbiol Ecol 87:121-132

65. Shigyo N, Umeki K, Hirao T (2019) Plant functional diversity and soil properties control elevational diversity gradients of soil bacteria. FEMS Microbiol Ecol 95:fiz025

66. Wang S, Bao X, Feng K et al (2021) Warming-driven migration of core microbiota indicates soil property changes at continental scale. Sci Bull 01:021

67. Ma M, Collins SL, Du G (2020) Direct and indirect effects of temperature and precipitation on alpine seed banks in the Tibetan Plateau. Ecol Appl 30:e02096

68. Peters MK, Hemp A, Appelhans T (2016) Predictors of elevational biodiversity gradients change from single taxa to the multi-taxa community level. Nat Commun 7:3736

69. Jenerette GD, Scott RL, Huxman TE (2008) Whole ecosystem metabolic pulses following precipitation events. Funct Ecol 22:924-930

70. Chen Q, Niu B, Hu Y et al (2020) Warming and increased precipitation indirectly affect the composition and turnover of labile-fraction soil organic matter by directly affecting vegetation and microorganisms. Sci Total Environ 714:136787

71. Rengel Z (2011) Soil pH, Soil Health and Climate Change. Springer-Verlag, Berlin

72. Shen $\mathrm{C}$, Xiong J, Zhang $\mathrm{H}$ et al (2013) Soil pH drives the spatial distribution of bacterial communities along elevation on Changbai Mountain. Soil Biol Biochem 57:204-211

73. Shen C, Liang W, Shi Y et al (2014) Contrasting elevational diversity patterns between eukaryotic soil microbes and plants. Ecology 95:3190-3202

74. Siciliano SD, Palmer AS, Winsley T et al (2014) Soil fertility is associated with fungal and bacterial richness, whereas $\mathrm{pH}$ is associated with community composition in polar soil microbial communities. Soil Biol Biochem 78:10-20

75. Barcenas-Moreno G, Baath E, Rousk J (2016) Functional implications of the pH-trait distribution of the microbial community in a re-inoculation experiment across a pH gradient. Soil Biol Biochem 93:69-78

76. Bates ST, Berg-Lyons D, Caporaso JG et al (2011) Examining the global distribution of dominant archaeal populations in soil. ISME J 5:908-917

77. Prescott CE, Grayston SJ (2013) Tree species influence on microbial communities in litter and soil: current knowledge and research needs. For Ecol Manag 309:19-27 
78. Leff JW, Bardgett RD, Wilkinson A et al (2018) Predicting the structure of soil communities from plant community taxonomy, phylogeny, and traits. ISME J 12:1794-1805

79. Wang J, Wang Y, He N et al (2020) Plant functional traits regulate soil bacterial diversity across temperate deserts. Sci Total Environ 715:136976

80. Delgado-Baquerizo M, Fry EL, Eldridge DJ et al (2018) Plant attributes explain the distribution of soil microbial communities in two contrasting regions of the globe. New Phytol 219:574-587

81. Vetaas OR, Paudel KP, Christensen M (2019) Principal factors controlling biodiversity along an elevation gradient: water, energy and their interaction. J Biogeogr 46:1652-1663

82. Santonja M, Rancon A, Fromin N et al (2017) Plant litter diversity increases microbial abundance, fungal diversity, and carbon and nitrogen cycling in a Mediterranean shrubland. Soil Biol Biochem 111:124-134

83. Delgado-Baquerizo M, Reich PB, Khachane AN et al (2017) It is elemental: soil nutrient stoichiometry drives bacterial diversity. Environ Microbiol 19:1176-1188

84. Dini-Andreote F, Stegen JC, van Elsas JD et al (2015) Disentangling mechanisms that mediate the balance between stochastic and deterministic processes in microbial succession. Proc Natl Acad Sci USA 112:E1326-E1332

85. Bryant JA, Lamanna C, Morlon $\mathrm{H}$ et al (2008) Microbes on mountainsides: contrasting elevational patterns of bacterial and plant diversity. Proc Natl Acad Sci USA 105:11505-11511

\section{Figures}



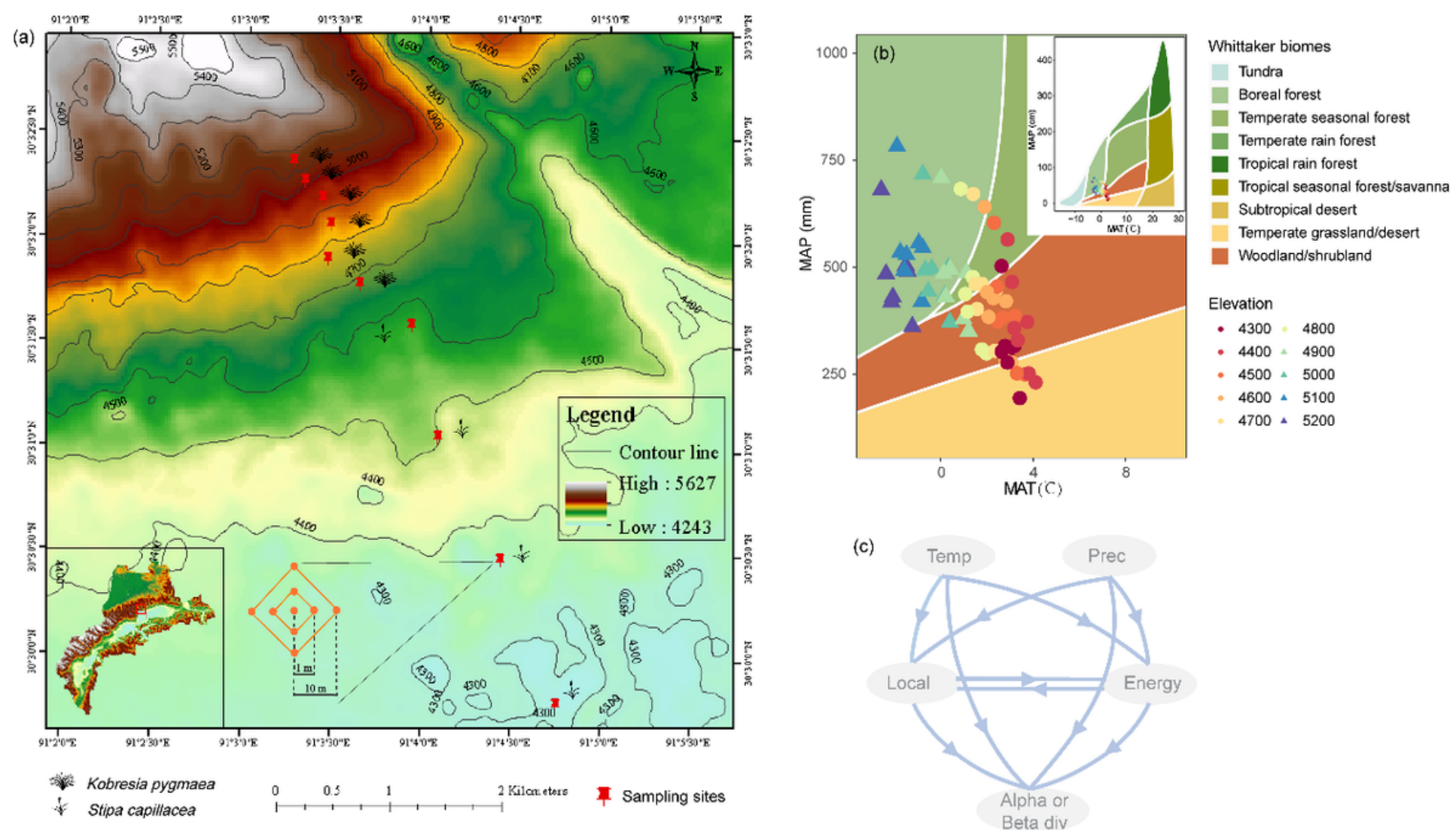

\section{Figure 1}

Sampling maps, Whittaker biome of sampling sites and hypothesized mechanisms for microbial communities. (a) Sampling sites with a nested design on Nyainqentanglha Mountain, Tibet. This elevational gradient extends across the alpine steppe and meadow ecosystems. (b) The mean annual temperature (MAT) and mean annual precipitation (MAP) of the sampling sites were projected onto the Whittaker biome plot. (c) Conceptual model showing hypothesized relationships among temperature, precipitation, local and energy variables and microbial diversity. The abbreviations for the environmental variables and their groups are listed in Table S4. Alpha or Beta div: microbial alpha or beta diversity. 

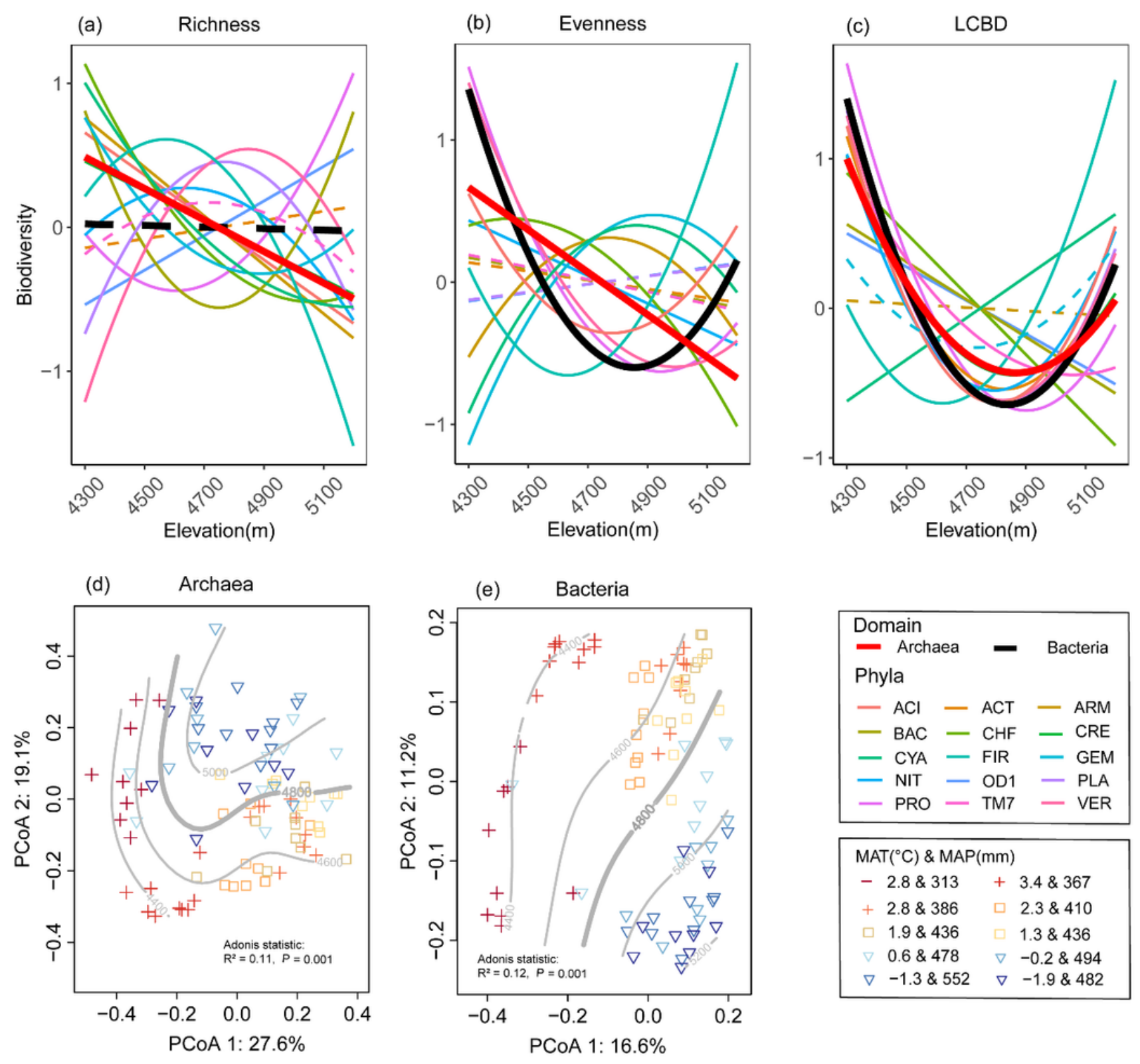

\section{Figure 2}

Elevational patterns in microbial community biodiversity. Species richness (a), evenness (b) and the local contribution to beta diversity (LCBD, c) were considered in the archaeal or bacterial communities and their phyla. The abbreviations for the phyla are listed in Table S2. A linear or quadratic model was selected based on the lower value of Akaike's information criterion. The biodiversity trends of the whole archaeal and bacterial communities are presented with bold red and black lines, respectively, and the trends of phylum biodiversity are shown with fine coloured lines. The solid and dotted lines represent significant ( $P$ 
$\llbracket 0.05)$ and nonsignificant $(P \otimes 0.05)$ results, respectively. Principal coordinate analysis (PCoA) plots of the composition of archaea (d) and bacteria (e). MAT and MAP of the sampling sites are shown in PCoA plots. The contours in grey indicate linear relationships between PCoA ordination values and elevations. ACl: Acidobacteria; ACT: Actinobacteria; ARM: Armatimonadetes; BAC: Bacteroidetes; CHF: Chloroflexi; CRE: Crenarchaeota; CYA: Cyanobacteria; FIR: Firmicutes; GEM: Gemmatimonadetes; NIT: Nitrospirae; PLA: Planctomycetes; PRO: Proteobacteria; VER: Verrucomicrobia.
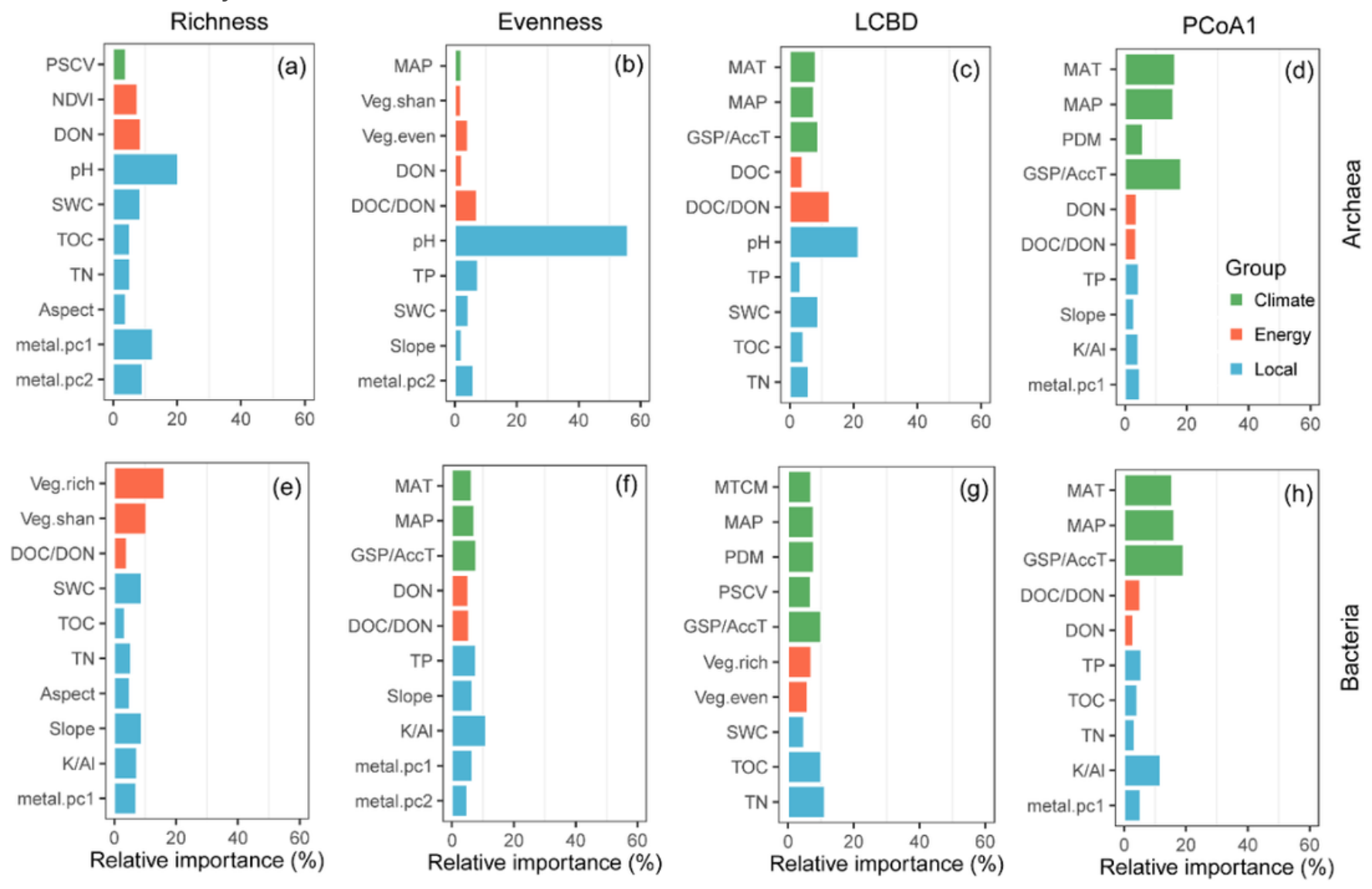

Figure 3

Relative importance of environmental variables for archaeal and bacterial richness $(a, e)$, evenness $(b, f)$, $\operatorname{LCBD}(c, g)$ and PCoA1 $(d, h)$. Relative importance was determined through random forest analysis including three groups of predictors: climate, energy and local variables. The abbreviations for the environmental variables and their groups are listed in Table S4. 

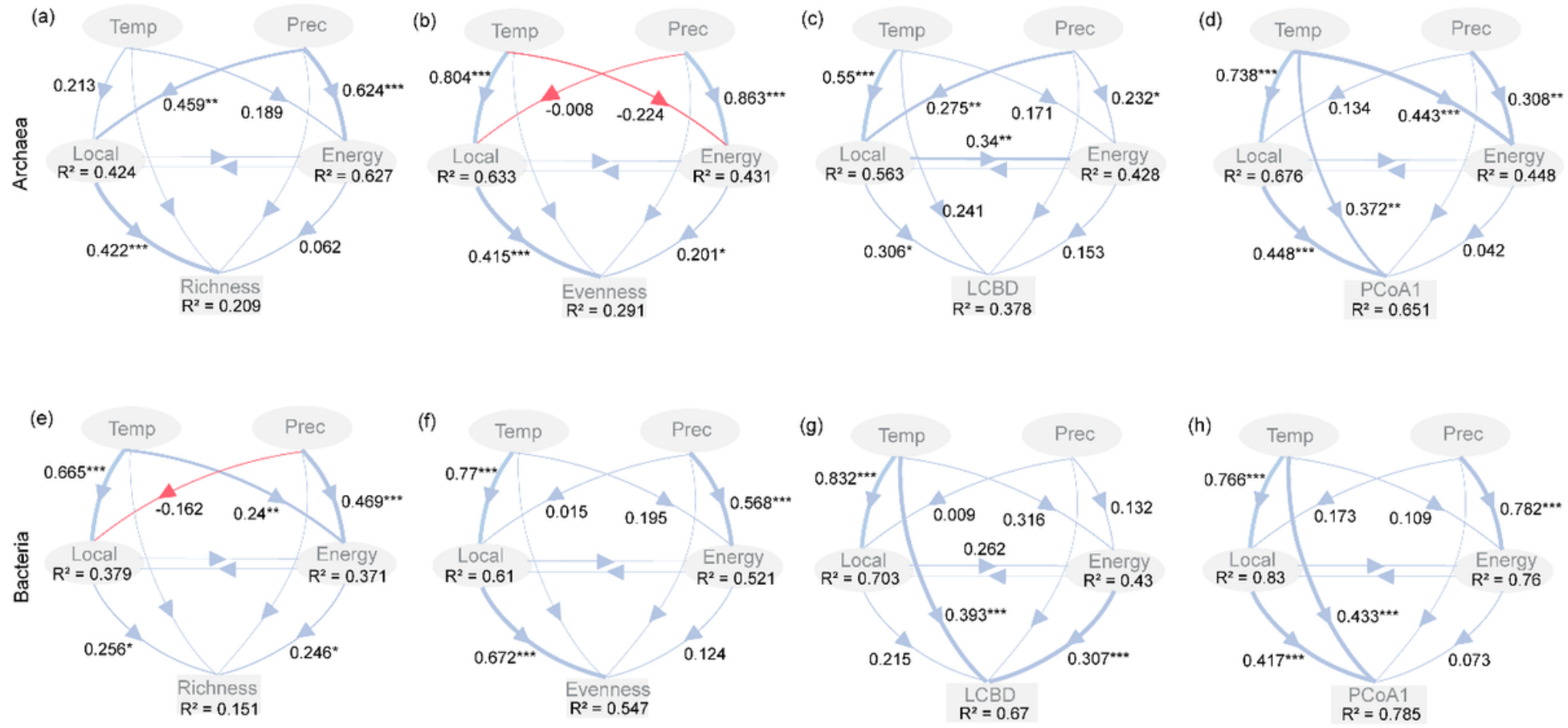

\section{Figure 4}

Structural equation models of archaeal and bacterial richness (a, e), evenness (b, f), LCBD (c, g) and PCoA1 $(d, h)$. The environmental variables included four groups of predictors: temperature, precipitation, energy and local variables. R2 denotes the proportion of variance explained for endogenous variables. Blue and red arrows indicate positive and negative relationships, respectively. Arrow widths and accompanying numbers represent the standardized path coefficients of the modelled relationships ( ${ }^{*} \mathrm{P}$ $\left.0.05,{ }^{*} \mathrm{P}<0.01,{ }^{\star} * \star \mathrm{P}<0.001\right)$. Composite and observed variables are indicated in ovals and rectangles, respectively. More details on the model fit are summarized in Tables S5-S6. The standardized total effects are listed in Fig. S19 and Table S7.

\section{Supplementary Files}

This is a list of supplementary files associated with this preprint. Click to download.

- SupplementaryMaterial.docx 